\title{
Normas Chilenas para el MACI: Una Integración de Criterios Categoriales y Dimensionales ${ }^{1}$
}

\section{Chilean Norms for MACI: An Integration of Categorical and Dimensional Criteria}

\author{
Eugenia V. Vinet \\ Universidad de La Frontera, Chile \\ María Forns i Santacana \\ Universidad de Barcelona, España \\ (Rec: 11 junio 2008 Acep: 24 de noviembre 2008)
}

\begin{abstract}
Resumen
Este estudio desarrolló normas chilenas para el Inventario Clínico para Adolescentes de Millon (MACI) utilizando una muestra de baremación diferenciada por sexo y dicotomizada en dos grupos, no-consultantes (200 hombres y 206 mujeres) y consultantes (212 hombres y 189 mujeres), que respondió el MACI y el MMPI-A. Con los datos MACI se desarrolló un conjunto de análisis estadísticos de validez diagnóstica en etapas sucesivas que dio lugar a la categorización de las escalas MACI en Acordes con la Teoría (AT), ContraTeóricas (CT) y No-Diferenciadoras, y a la determinación de puntajes de corte entre funcionamiento sano y alterado. Para las escalas AT y CT se diseñó una escala de baremación de 100 puntos cuyo puntaje central (PT 50) correspondió al puntaje de corte de cada escala. Las escalas ND fueron baremadas en percentiles. La discusión de resultados analiza aspectos psicométricos y de evaluación clínica considerando el contexto latinoamericano y la integración de estos resultados en el modelo teórico de Millon.

Palabras clave: MACI, baremos, evaluación psicológica clínica, adolescentes.
\end{abstract}

\begin{abstract}
This study aimed at building Chilean normative criteria for the Millon Adolescent Clinical Inventory (MACI) using a normative sample divided by sex, and into a non-consulting group (200 males and 206 females), and a clinical group (212 males and 189 females) that answered the MACI and the MMPI-A. Using MACI scales data, a set of statistical analysis for diagnostic validity were run through successive stages. Results indicated that MACI scales could be classified into Agreement with Theory (AT), Counter-Theory (CT); and Non-Discriminative (ND) scales; also a cutting point score between healthy functioning and disordered functioning was determined. To build norms for the AT and CT scales, a 100-point measuring scale that had the cutting-point score as the central score (PT 50) was used. Percentile norms were built for the ND scales. Discussion refers to psychometric and clinical assessment features attending to Latin-American cultural context and their integration into the Millon's theoretical model.
\end{abstract}

Key words: MACI, norms, clinical psychological assessment, adolescents.

\footnotetext{
El trabajo que da origen a este artículo recibió financiamiento de la Dirección de Investigación de la Universidad de La Frontera a través del Proyecto DIUFRO 120612. Correo electrónico de contacto: evinet@ufro.cl
} 


\section{Introducción}

Este artículo presenta los aspectos más relevantes de un extenso estudio orientado a desarrollar baremos chilenos para el Inventario Clínico para Adolescentes de Millon (MACI; Millon, 1993), llenando un vacío presente en la comunidad psicológica nacional al ofrecer un instrumento idóneo para la evaluación de adolescentes, con normas apropiadas para trabajar en los diversos ámbitos donde se requiera una evaluación clínica de la personalidad adolescente.

El MACI es un autoinforme de construcción racional, que evalúa estilos de personalidad, preocupaciones psicológicas y psicopatología en adolescentes. Los estilos de personalidad son estilos de funcionamiento psicológico relativamente estables que surgen a través del desarrollo infantil y se estabilizan en la adolescencia como los precursores de los estilos de personalidad adulta. Las preocupaciones psicológicas se relacionan con aspectos significativos del contexto de desarrollo del adolescente que pueden causarle preocupación, incluyen aspectos vinculados al desarrollo de la identidad, la corporalidad, la sexualidad y las relaciones sociales con los pares, la familia y la sociedad mayor. El área psicopatológica incluye los trastornos afectivos y de la adaptación social que tienen mayor prevalencia en la adolescencia.

El MACI fue desarrollado en Estados Unidos y actualmente es el autoinforme más usado en la evaluación clínica de adolescentes (Camara, Nathan \& Puente, 2000), está asociado a las clasificaciones de trastornos de la personalidad del DSM-IV (American Psychiatric Association, 1995), permite la identificación y comprensión de una amplia gama de dificultades psicológicas propias de los adolescentes y su simplicidad y brevedad (160 ítems con formato VerdaderoFalso) facilitan su uso en los ámbitos clínico y forense. Un gran número de investigaciones ha documentado su capacidad para describir el funcionamiento psicológico de los y las adolescentes, diferenciar y caracterizar las alteraciones psicopatológicas más prevalentes en la adolescencia y caracterizar a jóvenes de diferentes grupos poblacionales con problemas clínicos y de desadaptación social.

Este instrumento posee, además de la versión original en inglés (Millon, 1993), una versión en español para sujetos hispanos de Estados Unidos, una adaptación española con normas propias (TEA, 2004), y tres versiones en español desarrolladas en Argentina (Casullo, Góngora \& Castro, 1998), Perú (Iza, 2002), y Chile (Vinet et al. 1999a).

La versión chilena del MACI (Vinet et al. 1999a), desarrollada por un equipo bilingüe que utilizó el enfoque de comité y descentramiento (ver detalles en Vinet \& Alarcón, 2003a), ha dado origen a varios estudios a nivel nacional. Estos estudios señalan que las escalas del MACI poseen niveles aceptables de confiabilidad en diferentes grupos de jóvenes incluidos adolescentes de población general, con problemas clínicos y con problemas de adaptación social (Vinet, González \& Alarcón, 2002). Además, muestran que el instrumento posee una buena capacidad de discriminación entre adolescentes normales y clínicos (Vinet \& Alarcón, 2003a), y con problemas de desadaptación social (Alarcón, Vinet \& Salvo, 2005); que permite caracterizar diferencialmente a jóvenes con vulnerabilidad psicológica (Pérez, Díaz \& Vinet, 2005), a adolescentes en riesgo de trastornos psicopatológicos (Vinet, Salvo \& Forns, 2005) y a grupos con trastornos clínicos específicos en el ámbito de la depresión (Díaz, 2003) y de la drogadicción (Faundez, 2007). Con respecto a criterios externos, las escalas del MACI han presentado buenos niveles de correlación con escalas del Inventario Multifásico de la Personalidad de Minnesota para Adolescentes (MMPI-A; Butcher et al., 1992) que miden constructos similares (Vinet et al., 2004).

En todos los estudios chilenos mencionados los resultados del MACI han sido expresados en puntuaciones directas debido a la ausencia de baremos nacionales y porque, además, existen dudas sobre su aplicabilidad a poblaciones no-clínicas y a poblaciones procedentes de contextos culturales latinoamericanos. Estas dudas surgen, en primer lugar, porque Millon (1993) plantea que el MACI ha sido desarrollado para trabajar exclusivamente con poblaciones con trastornos clínicos y, en segundo lugar, porque la población latinoamericana no está representada en las normas del instrumento.

$\mathrm{Al}$ respecto debe señalarse que las normas originales del MACI, y también las normas españolas, están orientadas a la evaluación exclusiva de poblaciones clínicas y presentan sus puntuaciones en puntajes de Tasa Base, los cuales están asociados a la prevalencia que poseen, en las muestras clínicas de estandarización, las diversas características dimensionadas por el instrumento. Sin embargo, independientemente de su orientación clínica, el MACI ha sido aplicado en poblaciones no-clínicas en una gran diversidad de estudios que incluyen los procesos de adaptación del test a contextos socioculturales y lingüísticos diferentes del original (Casullo et al., 1998; Vinet \& Alarcón, 2003a), las relaciones entre estilos de personalidad no patológicos y otras variables de interés (Casullo \& Castro, 2002; Meeker, 2002), el screening psicopatológico en población general (Casullo \& Castro, 2002; Vinet et al., 2005; Wasserman, 2001), y la caracterización de jóvenes en situación de vulnerabilidad psicológica y social (Pérez et al., 2005).

El cuestionamiento sobre la aplicabilidad del test en contextos culturales latinoamericanos ha sido planteado en Estados Unidos al trabajar con sujetos hispanos (Blumentritt, Angle \& Brown, 2004; Blumentritt \& Wilson, 2004). En ellos se ha observado que, de modo contrario a lo esperado teóricamente, puntuaciones altas en determinadas escalas (Dramatizador, Egoísta y Conformista) están asociadas en este grupo cultural a aspectos más saludables que patológicos, estableciéndose un vínculo 
entre características de la cultura latina y rasgos personales asociados al respeto de las normas, el cuidado del grupo de pares y la sociabilidad (Blumentritt et al., 2004). En estudios chilenos y argentinos donde se ha evaluado a grupos de población general y grupos clínicos (Casullo \& Castro, 2002; Vinet \& Alarcón, 2003a), se ha observado que, en las mismas escalas señaladas por Blumentritt et al. (2004), los puntajes más altos se presentan en los grupos de población general planteándose la asociación entre los puntajes de estas escalas y la salud mental.

Este hallazgo ha sido estudiado por Vinet y Forns (2006) en adolescentes chilenos. En ese estudio las escalas correspondientes a los patrones Dramatizador, Egoísta y Conformista y la correspondiente a la preocupación expresada Incomodidad Sexual presentan puntajes mayores en un grupo de población general al compararlo con un grupo de adolescentes con problemas clínicos; además, se señala que las escalas Sumiso, Insensibilidad Social, Predisposición Delictiva y Sentimientos de Ansiedad no son capaces de diferenciar entre estos dos grupos de adolescentes. Las autoras hacen comprensibles estos resultados, que indican la existencia de tres grupos de escalas con funcionamientos diferentes, al analizar el rol que podría jugar la cultura a la que pertenecen los sujetos en sus respuestas al test.

Estos antecedentes permiten plantear que una adecuada adaptación del MACI para ser utilizado en Chile y en poblaciones normales y clínicas, debería resolver los interrogantes respecto del funcionamiento diferencial de determinadas escalas en el contexto cultural chileno y desarrollar un estudio de baremación ad-hoc que considere tanto la población general como la población clínica.

Así, el objetivo de este estudio es la evaluación de las escalas del MACI para diferenciar entre población general y clínica en nuestro contexto cultural y el desarrollo de una escala de baremación cuya característica principal será un puntaje de corte central que permita diferenciar entre los adolescentes procedentes de población general y aquellos procedentes de población clínica. Consecuentemente, los baremos que se desarrollarán serán diferentes de la baremación original del MACI en los siguientes tres aspectos: (1) considerarán las diferencias entre el contexto sociocultural de origen (Estados Unidos) y el contexto sociocultural de nuestro país, (2) extenderán el uso del MACI desde la población clínica a poblaciones no-clínicas, favoreciendo la detección de casos en riesgo de alteraciones de la salud mental y (3) propondrán una escala de medida para la baremación que es diferente de las escalas tradicionales expresadas en puntajes $\mathrm{T}$ o percentiles y también diferente de la escala de baremación tradicional del MACI expresada en puntuaciones de Tasa Base.

\section{Método}

\section{Población y muestra}

La muestra utilizada está compuesta por dos grandes grupos. Uno proviene de la población general de adolescentes y representa las características generales de los adolescentes chilenos, ellos son los adolescentes "no-consultantes", sin patología evidente. El segundo grupo proviene de instituciones que atienden a adolescentes en riesgo o con trastornos de la salud mental y/o de la adaptación psicosocial, ellos representan a los adolescentes "consultantes" afectados por problemas de salud mental.

Esta muestra luego de un proceso de reducción por etapas quedó constituida por 807 adolescentes que cumplieron los siguientes criterios de validez del MACI: (a) ausencia de ítems omitidos o con dobles marcas. (b) puntuación directa de la escala X (Sinceridad) mayor o igual a 201 e inferior o igual a 589 y (c) puntuación en la escala V (Validez) igual o inferior a 1. Además la muestra cumplió también con los criterios de validez para el MMPI-A propuestos por Lucio, Ampudia y Durán (1998) para la versión mexicana del instrumento.

\section{Instrumentos}

El estudio completo de baremación incluye dos instrumentos: el MACI, instrumento para el cual se desarrollarán los baremos, y el MMPI-A (Butcher et al., 1992), instrumento que es utilizado como criterio externo para validar los puntajes de corte del MACI, aspecto logrado en el trabajo realizado pero que no se reportará específicamente en el presente artículo. Consecuentemente con esta decisión, en el presente artículo sólo se describen las características del MACI en aquellos aspectos no señalados explícitamente en los antecedentes presentados previamente.

Inventario Clínico para Adolescentes de Millon (MACI; Millon, 1993). Es un instrumento de construcción racional que sigue los modelos de aprendizaje biosocial y evolutivo de la personalidad desarrollados por Millon $(1969,1990)$ y que consta de 160 ítems con formato Verdadero-Falso. Los ítems se organizan en 31 escalas, 27 de ellas con significado clínico, estas son doce escalas de Patrones de Personalidad, ocho escalas de Preocupaciones Expresadas y siete escalas de Síndromes Clínicos. Las escalas no clínicas son tres escalas de control denominadas Escalas Modificadoras y una escala de validez (V) de sólo dos ítems. La denominación de cada escala, según la versión chilena, aparece en la primera columna de la Tabla 3 y siguientes.

La fiabilidad de las escalas ha sido probada en diversos estudios. En muestras chilenas los índices de consistencia interna obtenidos a través del coeficiente $A l f a$ de Cronbach son similares a los obtenidos por Millon (1993) y oscilan entre .54 (D) y .90 (8B) en las muestras de no-pacientes 
y entre .51 (D) y .91 (8B y B) en las muestras de sujetos con problemas psicológicos, con medianas de .79 y .81 respectivamente (Vinet \& Alarcón, 2003a).

El instrumento ha demostrado ser una prueba válida, con una adecuada capacidad de discriminación entre adolescentes normales y grupos con problemas psicológicos (Vinet \& Alarcón, 2003a) y con buenas posibilidades para caracterizar psicológicamente a diversos grupos en riesgo y con problemas clínicos y de desadaptación social (Alarcón et al. 2005; Díaz, 2003; Faúndez, 2007; Vinet et al. 2005).

El MACI cuenta en la actualidad con dos grupos de normas, estadounidenses y españolas, que están orientadas a evaluar diferenciadamente a hombres y mujeres entre 13 y 19 años; estas normas se expresan en puntajes de Tasa Base, los cuales están asociados a tasas de prevalencia de trastornos mentales. La ausencia de baremos para población chilena impulsa el trabajo que aquí se presenta.

\section{Procedimiento y análisis de datos}

El desarrollo de baremos involucró una serie de etapas sucesivas que se desarrollaron a partir de la obtención y caracterización de la muestra de baremación.

Para constituir la muestra, un extenso grupo de adolescentes de 13 a 19 años, procedentes de diversos centros educacionales y de centros de atención de adolescentes con problemas psicológicos, fue invitado a participar en un proceso de evaluación que buscaba conocer las características psicológicas de los adolescentes chilenos. Las dos pruebas utilizadas fueron presentadas en los centros educacionales (no-consultantes) y los centros de atención de adolescentes con problemas psicológicos (consultantes) como las herramientas que permitirían acceder de modo sistemático al funcionamiento psicológico de los adolescentes. Todos los adolescentes proporcionaron datos sociodemográficos básicos y respondieron primero el MACI y posteriormente el MMPI-A.

La muestra de baremación quedó definida a partir de los requisitos de validez de los dos instrumentos y se la caracterizó utilizando la información sociodemográfica e información adicional obtenida en entrevistas complementarias.

En las etapas siguientes, el aspecto más relevante fue poder determinar, en cada escala y separadamente para cada sexo, un puntaje que permitiera discriminar entre no-consultantes y consultantes, optimizando la capacidad de screening de la prueba. Este puntaje discriminativo o puntaje de corte que permitirá clasificar el funcionamiento psicológico de los sujetos evaluados a través del MACI en dos categorías - perteneciente a un grupo con funcionamiento sano (no-consultante) o perteneciente a un grupo con funcionamiento alterado (consultante)- surge a través de análisis empíricos que siguen la metodología de las pruebas diagnósticas (Hsu, 2002; Pita \& Pértegas, 2003).
Con este objetivo, en primer lugar, se categorizaron las escalas clínicas del MACI según su capacidad de discriminación entre no-consultantes y consultantes y la dirección ascendente o descendente de la psicopatología en la puntuación de cada escala. Para ello se utilizó un conjunto de indicadores estadísticos conocidos como "estadísticos de validez diagnóstica" (diagnostic validity statistics, Hsu, 2002) que incluyen los índices de magnitud del efecto de Cohen $(d)$ y una medida de no-sobreposición (nonoverlap) de distribuciones de dos grupos $\left(U_{1}\right)$ también desarrollada por Cohen (1988); ellos complementan la información entregada por la clásica prueba $t$ de Student al informar sobre la magnitud de las diferencias entre los grupos y el porcentaje de no-sobreposición entre las distribuciones de los grupos comparados.

En segundo lugar, se realizó para cada escala del MACI, un análisis de curva ROC utilizando el software Rockit 0.9B, Beta Versión, desarrollado por C. Metz (1998). Este análisis, cuyos resultados pueden presentarse gráficamente, permitió evaluar la capacidad de discriminación global de cada escala a través del estadístico Área Bajo la Curva $(A B C$ o $A z)$.

En tercer lugar, para cada escala con un poder discriminativo adecuado evaluado a través de los análisis precedentes, se determinó un puntaje de corte óptimo para diferenciar entre no-consultantes y consultantes utilizando los indicadores estadísticos de sensibilidad (S), especificidad (E) e índices complementarios, los cuales informan sobre el poder de discriminación de un puntaje particular. Cada puntaje de corte fue validado empíricamente a través su relación con el MMPI-A, aspecto que será tratado en otra publicación.

Una vez determinados los puntajes de corte, los baremos fueron desarrollados en una escala de medida trasformada de 100 puntos que ubica como punto central de la baremación de cada escala (puntaje 50) el puntaje de corte determinado previamente. Los otros puntajes fueron determinados mediante transformación lineal entre el mínimo y máximo posible en cada escala.

\section{Resultados}

Los resultados que se presentan a continuación dan cuenta de los aspectos esenciales de la baremación realizada: caracterización de la muestra de baremación, categorización de las escalas del MACI, determinación de los puntajes de corte y desarrollo de baremos.

\section{Caracterización de la muestra de baremación}

La muestra de baremación constituida por 807 adolescentes (406 no-consultantes y 401 consultantes) presenta la distribución que se expone en la Tabla 1 para las variables de grupo, sexo, edad y años de escolaridad. 
Análisis estadísticos indican que los grupos de noconsultantes y consultantes son equivalentes en su distribución por sexo $\left[\chi^{2}(1, \mathrm{n}=807)=1.05, p=.305\right]$ y por edad $\left(M_{\text {Nocon }}=15.86\right.$ años, $D T=1.61 ; M_{\text {Con }}=15.78$ años, $D T=$ $1.66 ; t=0.69, p=.493$ ) y que los no-consultantes presentan un nivel de escolaridad significativamente mayor que los consultantes $\left(M_{\text {Nocon }}=10.59\right.$ años, $D T=1.57 ; M_{\text {Con }}=9.35$ años, $D T=1.94, t=9.96, p<.001)$.
Los no-consultantes proceden de la zona Centro-Sur del país, específicamente de las ciudades de Concepción y Temuco. La gran mayoría de ellos está cursando su ciclo de Enseñanza Media (76.6\% de los hombres y $81.6 \%$ de las mujeres) encontrándose el resto distribuido en los últimos años de la Enseñanza Básica (10\% y 8.7\% de hombres y mujeres respectivamente) e inicios de la Enseñanza Superior (13.4\% de hombres y $9.7 \%$ de mujeres).

Tabla 1. Distribución de los grupos de no-consultantes y consultantes de la muestra de baremación por sexo, edad y años de escolaridad.

\begin{tabular}{|c|c|c|c|c|c|c|c|}
\hline \multirow{2}{*}{ Variables } & \multicolumn{3}{|c|}{ No-consultantes } & \multicolumn{3}{|c|}{ Consultantes } & \multirow{2}{*}{ Totales } \\
\hline & Hombres & Mujeres & Total & Hombres & Mujeres & Total & \\
\hline \multicolumn{8}{|l|}{ Edad } \\
\hline 13 & 19 & 18 & 37 & 25 & 24 & 49 & 86 \\
\hline 14 & 31 & 31 & 62 & 26 & 31 & 57 & 119 \\
\hline 15 & 27 & 31 & 58 & 32 & 24 & 56 & 114 \\
\hline 16 & 42 & 47 & 89 & 47 & 35 & 82 & 171 \\
\hline 17 & 48 & 51 & 99 & 61 & 39 & 100 & 199 \\
\hline 18 & 24 & 22 & 46 & 16 & 26 & 42 & 86 \\
\hline 19 & 9 & 6 & 15 & 5 & 10 & 15 & 30 \\
\hline \multicolumn{8}{|c|}{ Años de Escolaridad } \\
\hline 6 o menos & 1 & 0 & 1 & 16 & 8 & 24 & 25 \\
\hline 7 & 3 & 6 & 9 & 28 & 14 & 42 & 51 \\
\hline 8 & 15 & 12 & 27 & 42 & 29 & 71 & 98 \\
\hline 9 & 40 & 38 & 78 & 40 & 29 & 69 & 147 \\
\hline 10 & 30 & 34 & 64 & 37 & 40 & 77 & 141 \\
\hline 11 & 48 & 49 & 97 & 29 & 31 & 60 & 157 \\
\hline 12 & 36 & 47 & 83 & 15 & 29 & 44 & 127 \\
\hline 13 o más & 27 & 20 & 47 & 5 & 8 & 13 & 60 \\
\hline Omitidos & -- & -- & -- & -- & 1 & 1 & 1 \\
\hline Totales & 200 & 206 & 406 & 212 & 189 & 401 & 807 \\
\hline
\end{tabular}

Tabla 2. Categorización de los consultantes según instituciones de procedencia.

\begin{tabular}{|c|c|c|}
\hline Institución & $\mathrm{f}$ & $\%$ \\
\hline \multicolumn{3}{|l|}{ Centros de atención clínica } \\
\hline Centro de salud mental público, ambulatorio & 224 & 55.9 \\
\hline Consulta o centro privado de salud mental, ambulatorio & 37 & 9.2 \\
\hline Colegio, con atención psicológica ambulatoria & 43 & 10.7 \\
\hline Clínica u hospital psiquiátrico, interno. & 5 & 1.2 \\
\hline Subtotal & 309 & 77.1 \\
\hline \multicolumn{3}{|l|}{ Centros de atención judicial } \\
\hline Hogares para adolescentes con problemas psicosociales & 16 & 4.0 \\
\hline Centro de evaluación judicial, ambulatorio & 8 & 2.0 \\
\hline Centro rehabilitación judicial, ambulatorio & 21 & 5.2 \\
\hline Centro de rehabilitación judicial, interno & 47 & 11.7 \\
\hline Subtotal & 92 & 22.9 \\
\hline Total & 401 & 100.0 \\
\hline
\end{tabular}


Los consultantes proceden en su gran mayoría (94\%) de ciudades del Centro-Sur del país, entre las que se incluyen, además de Temuco y Concepción, tres ciudades de menor tamaño. El 6\% restante procede de la ciudad de Santiago. Su menor nivel educacional está asociado a mayor variabilidad y a que el $14.7 \%$ de ellos ha abandonado el sistema escolar. Además, cerca del $75 \%$ de los consultantes procede de centros clínicos donde reciben atención por problemas de salud mental, en tanto que el $25 \%$ restante procede de centros vinculados al sistema judicial en los que reciben atención por problemas psicosociales y de adaptación social. La Tabla 2 muestra en detalle la procedencia de los consultantes.

\section{Categorización de las escalas clínicas del MACI}

Con el objeto de evaluar la capacidad de discriminación de las escalas clínicas del MACI entre no-consultantes y consultantes, de determinar la dirección ascendente o descendente de las puntuaciones de psicopatología y poder clasificar a las escalas en los grupos encontrados por Vinet y Forns (2006), se realizaron dos grupos de análisis complementarios trabajando separadamente con hombres y mujeres: (a) cómputo y comparación de las medias de no-consultantes y consultantes, para cada una de las escalas clínicas del MACI, incluyendo los estadísticos de magnitud del efecto $(d)$ y no-sobreposición de las distribuciones $\left(U_{1}\right)$

Tabla 3. Estadísticos descriptivos, contraste de grupos y evaluación de la capacidad de discriminación en las escalas MACI de los hombres de la muestra de baremación.

\begin{tabular}{|c|c|c|c|c|c|c|c|c|c|c|c|}
\hline & & Cons & intes & Cons & tantes & & & & & & \\
\hline & Escalas & $\mathrm{M}$ & DT & $\mathrm{M}$ & DT & & & $\mathrm{d}$ & $\mathrm{U}_{1}$ & & $\mathrm{o} \mathrm{Az}$ \\
\hline 1 & Introvertido & 20,93 & 7,28 & 27,82 & 9,79 & $-8,05$ & $* * *$ & $-0,80$ & 47,2 & .70 & $* * *$ \\
\hline $2 \mathrm{~A}$ & Inhibido & 16,73 & 6,98 & 24,07 & 10,35 & $-8,38$ & $* * *$ & $-0,83$ & 48,6 & .72 & $* * *$ \\
\hline $2 \mathrm{~B}$ & Afligido & 8,09 & 5,70 & 18,67 & 10,01 & $-13,16$ & $* * *$ & $-1,29$ & 65,1 & .81 & $* * *$ \\
\hline 3 & Sumiso & 48,56 & 7,41 & 46,03 & 10,84 & 2,75 & $* *$ & 0,27 & 19,5 & .59 & * \\
\hline 4 & Dramatizador & 41,79 & 7,39 & 38,86 & 9,39 & 3,52 & $* * *$ & 0,35 & 24,2 & .59 & $* *$ \\
\hline 5 & Egoísta & 38,86 & 7,55 & 34,13 & 8,77 & 5,81 & $* * *$ & 0,58 & 37,0 & .66 & $* * *$ \\
\hline $6 \mathrm{~A}$ & Trasgresor & 26,94 & 8,47 & 37,83 & 11,72 & $-10,74$ & $* * *$ & $-1,06$ & 57,6 & .77 & $* * *$ \\
\hline $6 \mathrm{~B}$ & Poderoso & 9,27 & 5,54 & 14,85 & 8,40 & $-7,97$ & $* * *$ & $-0,78$ & 46,6 & .70 & $* * *$ \\
\hline 7 & Conformista & 51,01 & 6,33 & 41,33 & 10,30 & 11,51 & $* * *$ & 1,13 & 59,8 & .79 & $* * *$ \\
\hline $8 \mathrm{~A}$ & Oposicionista & 16,19 & 6,76 & 27,39 & 11,19 & $-12,31$ & $* * *$ & $-1,20$ & 62,3 & .80 & $* * *$ \\
\hline $8 \mathrm{~B}$ & Autodegradante & 12,14 & 8.00 & 26,10 & 12,88 & $-13,14$ & $* * *$ & $-1,30$ & 65,1 & .82 & $* * *$ \\
\hline 9 & Tendencia Limítrofe & 8,90 & 4,76 & 17,04 & 8,69 & $-11,88$ & $* * *$ & $-1,15$ & 60,7 & .79 & $* * *$ \\
\hline A & Difusión de la Identidad & 11,35 & 5,38 & 19,00 & 7,98 & $-11,48$ & $* * *$ & $-1,12$ & 59,5 & .79 & $* * *$ \\
\hline B & Autodevaluación & 12,74 & 8,18 & 26,37 & 13,29 & $-12,56$ & $* * *$ & $-1,20$ & 62,2 & .81 & $* * *$ \\
\hline $\mathrm{C}$ & Desaprobación Corporal & 4,78 & 4,47 & 8,21 & 6,92 & $-5,99$ & $* * *$ & $-0,59$ & 37,4 & .66 & $* * *$ \\
\hline $\mathrm{D}$ & Incomodidad Sexual & 30,59 & 5,44 & 26,92 & 7,29 & 5,78 & $* * *$ & 0,57 & 36,5 & .65 & $* * *$ \\
\hline E & Inseguridad Grupal & 8,25 & 3,55 & 10,42 & 6,07 & $-4,45$ & $* * *$ & $-0,43$ & 29,3 & .59 & $* *$ \\
\hline $\mathrm{F}$ & Insensibilidad Social & 27,92 & 6,83 & 30,14 & 9,08 & $-2,82$ & $* *$ & $-0,28$ & 19,7 & .58 & $*$ \\
\hline G & Discordia Familiar & 12,89 & 4,21 & 18,38 & 7,05 & $-9,65$ & $* * *$ & $-0,94$ & 53,1 & .73 & $* * *$ \\
\hline $\mathrm{H}$ & Abuso Infantil & 3,79 & 3,27 & 8,82 & 4,90 & $-12,25$ & $* * *$ & $-1,20$ & 62,2 & .81 & $* * *$ \\
\hline AA & Disfunciones Alimentarias & 5,26 & 4,71 & 9,38 & 7,75 & $-6,51$ & $* * *$ & $-0,64$ & 40,1 & .67 & $* * *$ \\
\hline $\mathrm{BB}$ & Tend. al Abuso Sustancias & 10,61 & 6,82 & 22,39 & 12,49 & $-11,91$ & $* * *$ & $-1,16$ & 61,0 & .80 & $* * *$ \\
\hline $\mathrm{CC}$ & $\begin{array}{l}\text { Predispos. a } \quad 1 \text { a } \\
\text { Delincuencia }\end{array}$ & 23,27 & 4,98 & 27,87 & 8,35 & $-6,78$ & $* * *$ & $-0,67$ & 41,4 & .67 & $* * *$ \\
\hline DD & Tend. a la Impulsividad & 14,90 & 5,36 & 20,49 & 6,97 & $-9,12$ & $* * *$ & $-0,90$ & 51,4 & .73 & $* * *$ \\
\hline $\mathrm{EE}$ & Sentimientos de Ansiedad & 31,05 & 5,38 & 28,31 & 8,05 & 4,07 & $* * *$ & 0,40 & 27,3 & .61 & $* * *$ \\
\hline $\mathrm{FF}$ & Afecto Depresivo & 10,55 & 5,73 & 18,74 & 10,45 & $-9,92$ & $* * *$ & $-0,96$ & 54,1 & .74 & $* * *$ \\
\hline GG & Tendencia Suicida & 3,48 & 3,44 & 11,34 & 8,77 & $-12,07$ & $* * *$ & $-1,17$ & 61,2 & .81 & $* * *$ \\
\hline
\end{tabular}

$* * * p<.001 * * p<.01 * p<.05$ 
Tabla 4. Estadísticos descriptivos, contraste de grupos y evaluación de la capacidad de discriminación en las escalas MACI de las mujeres de la muestra de baremación.

\begin{tabular}{|c|c|c|c|c|c|c|c|c|c|c|c|}
\hline \multirow{2}{*}{\multicolumn{2}{|c|}{ Escalas }} & \multicolumn{2}{|c|}{$\begin{array}{c}\text { No } \\
\text { Consultantes }\end{array}$} & \multicolumn{2}{|c|}{ Consultantes } & \multirow{2}{*}{\multicolumn{2}{|c|}{ Prueba t }} & \multirow[b]{2}{*}{ d } & \multirow{3}{*}{$\mathrm{U}_{1}$} & \multirow{2}{*}{\multicolumn{2}{|c|}{$\mathrm{ABC}$ o $\mathrm{Az}$}} \\
\hline & & $\mathrm{M}$ & DT & $\mathrm{M}$ & DT & & & & & & \\
\hline 1 & Introvertido & 19,90 & 7,06 & 33,28 & 10,62 & $-14,46$ & $* * *$ & $-1,50$ & & .84 & $* * *$ \\
\hline $2 \mathrm{~A}$ & Inhibido & 19,80 & 7,37 & 33,07 & 11,68 & $-13,29$ & $* * *$ & $-1,37$ & 67,3 & .82 & $* * *$ \\
\hline $2 \mathrm{~B}$ & Afligido & 9,71 & 7,00 & 25,88 & 11,65 & $-16,47$ & $* * *$ & $-1,70$ & 75,3 & .87 & $* * *$ \\
\hline 3 & Sumiso & 54,97 & 7,23 & 52,05 & 10,50 & 3,17 & $* *$ & 0,33 & 22,9 & .58 & $*$ \\
\hline 4 & Dramatizador & 39,82 & 7,50 & 30,89 & 10,20 & 9,80 & $* * *$ & 1,00 & 55,5 & .76 & $* * *$ \\
\hline 5 & Egoísta & 34,61 & 8,76 & 26,82 & 9,82 & 8,28 & $* * *$ & 0,84 & 49,1 & .72 & $* * *$ \\
\hline $6 \mathrm{~A}$ & Trasgresor & 21,87 & 8,31 & 30,82 & 11,41 & $-8,80$ & $* * *$ & $-0,90$ & 51,6 & .73 & $* * *$ \\
\hline $6 \mathrm{~B}$ & Poderoso & 7,00 & 4,57 & 13,63 & 7,87 & $-9,98$ & $* * *$ & $-1,03$ & 56,3 & .76 & $* * *$ \\
\hline 7 & Conformista & 52,47 & 6,60 & 40,69 & 10,98 & 12,74 & $* * *$ & 1,31 & 65,6 & .82 & $* * *$ \\
\hline $8 \mathrm{~A}$ & Oposicionista & 14,92 & 7,87 & 30,45 & 12,07 & $-14,97$ & $* * *$ & $-1,54$ & 71,6 & .86 & $* * *$ \\
\hline $8 \mathrm{~B}$ & Autodegradante & 15,80 & 10,31 & 36,33 & 14,88 & $-15,65$ & $* * *$ & $-1,62$ & 73,5 & .86 & $* * *$ \\
\hline 9 & Tendencia Limítrofe & 10,05 & 6,54 & 21,70 & 9,52 & $-14,03$ & $* * *$ & $-1,44$ & 69,1 & .84 & $* * *$ \\
\hline A & Difusión de la Identidad & 11,82 & 6,62 & 21,96 & 9,18 & $-12,49$ & $* * *$ & $-1,28$ & 64,5 & .81 & $* * *$ \\
\hline $\mathrm{B}$ & Autodevaluación & 18,00 & 11,03 & 38,01 & 15,19 & $-14,80$ & $* * *$ & $-1,52$ & 71,1 & .85 & $* * *$ \\
\hline $\mathrm{C}$ & Desaprobación Corporal & 10,53 & 7,68 & 17,32 & 8,84 & $-8,11$ & $* * *$ & $-0,82$ & 48,4 & .72 & $* * *$ \\
\hline $\mathrm{D}$ & Incomodidad Sexual & 35,21 & 4,81 & 32,12 & 6,23 & 5,46 & $* * *$ & 0,56 & 36,0 & .64 & $* * *$ \\
\hline $\mathrm{E}$ & Inseguridad Grupal & 8,16 & 2,81 & 13,71 & 7,45 & $-9,63$ & $* * *$ & $-1,00$ & 55,4 & .73 & $* * *$ \\
\hline $\mathrm{F}$ & Insensibilidad Social & 21,70 & 6,74 & 21,67 & 8,91 & 0,04 & & 0,00 & 0,3 & .51 & \\
\hline G & Discordia Familiar & 13,33 & 5,35 & 20,88 & 8,09 & $-10,83$ & $* * *$ & $-1,11$ & 59,3 & .77 & $* * *$ \\
\hline $\mathrm{H}$ & Abuso Infantil & 5,52 & 4,64 & 13,74 & 7,31 & $-13,10$ & $* * *$ & $-1,36$ & 66,9 & .84 & $* * *$ \\
\hline AA & Disfunciones Alimentarias & 13,16 & 9,09 & 22,25 & 11,08 & $-8,86$ & $* * *$ & $-0,90$ & 51,6 & .73 & $* * *$ \\
\hline BB & Tend. al Abuso Sustancias & 7,44 & 5,61 & 17,40 & 10,93 & $-11,16$ & $* * *$ & $-1,16$ & 61,0 & .80 & $* * *$ \\
\hline $\mathrm{CC}$ & Predispos. a la Delincuencia & 18,42 & 4,18 & 17,99 & 7,11 & 0,72 & & 0,07 & 5,8 & .52 & \\
\hline DD & Tend. a la Impulsividad & 12,65 & 5,66 & 18,07 & 7,25 & $-8,22$ & $* * *$ & $-0,84$ & 49,0 & .72 & $* * *$ \\
\hline $\mathrm{EE}$ & Sentimientos de Ansiedad & 35,93 & 5,14 & 35,45 & 8,20 & 0,69 & & 0,07 & 5,5 & .52 & \\
\hline $\mathrm{FF}$ & Afecto Depresivo & 15,26 & 7,64 & 29,69 & 12,10 & $-14,03$ & $* * *$ & $-1,44$ & 69,1 & .83 & $* * *$ \\
\hline GG & Tendencia Suicida & 5,45 & 5,62 & 17,97 & 10,60 & $-14,43$ & $* * *$ & $-1,49$ & 70,5 & .86 & $* * *$ \\
\hline
\end{tabular}

$* * * p<.001 * * p<.01 * p<.05$

y (b) cómputo, a través de análisis de curvas ROC, de la capacidad global de cada escala para diferenciar entre noconsultantes y consultantes. Los resultados de este conjunto de análisis se presentan en la Tabla 3 para los hombres y en la Tabla 4 para las mujeres.

En estas tablas se puede observar que existe un gran grupo de escalas MACI que presentan diferencias altamente significativas (prueba $t$ con $p<.001$ ) entre no-consultantes y consultantes tanto en hombres como en mujeres. El estadístico $d$ indica que, en estas escalas, el tamaño de la diferencia entre no-consultantes y consultantes es moderado o alto (valores superiores o cercanos a 0.35 ) y el estadístico $U$, señala que el porcentaje de no-sobreposición entre las distribuciones de estas escalas tiene un valor cercano o superior al 50\%. El análisis ROC muestra que estas escalas presentan un área bajo la curva $(A z)$ altamente significativa $(p<.001)$ la cual avala la buena capacidad de discriminación global de estas escalas.

El cómputo de pruebas $t$ indica que existe un segundo grupo de escalas que también presenta diferencias significativas pero menores $(p<.01)$ entre los grupos. En los hombres esto sucede en las escalas Sumiso (3) e Insensibilidad Social $(\mathrm{F})$ y en las mujeres sólo en la escala Sumiso. En estos casos el estadístico $d$ tiene un valor cercano a 0.30 que indica un tamaño de la diferencia moderado a bajo. El estadístico $U_{1}$ para estas escalas muestra un porcentaje de no-sobreposición entre las distribuciones cercano al $20 \%$ y el estadístico $A z$ señala un área bajo la curva menor pero 
significativa $(p<.05)$, que indica un nivel de discriminación global relativamente bajo (cercano a .60) en estas escalas.

Las pruebas $t$ muestran un tercer grupo de escalas, presente sólo en las mujeres, en las cuales no existen diferencias significativas entre no-consultantes y consultantes. Esto sucede en las escalas Insensibilidad Social, Predisposición a la Delincuencia, y Sentimientos de Ansiedad (F, CC y EE respectivamente). En ellas tanto $d$ como $U_{1}$ tienen valores muy bajos y $A z$ (no significativo) indica un área bajo la curva muy cercana al área de no discriminación $(A z=.50)$.

Por otra parte, al revisar la dirección de las diferencias altas y moderadamente significativas se observa que, aunque en la gran mayoría de las escalas los puntajes medios mayores corresponden a los consultantes, existen escalas donde los puntajes medios mayores corresponden a los no-consultantes. Esto sucede en hombres y mujeres en las escalas Sumiso, Dramatizador, Egoísta, Conformista e Incomodidad Sexual (escalas 3, 4, 5, 7 y D) y también en la escala de Ansiedad (EE) en la muestra de hombres. Esta observación evidencia que hay escalas que no se comportan conforme a lo esperado teóricamente en el modelo de Millon sino que lo hacen de modo inverso o contra-teórico aunque poseen una capacidad de discriminación adecuada.

Tabla 5. Clasificación de las escalas según su categoría y poder de discriminación entre no consultantes y consultantes.

\begin{tabular}{|c|c|c|c|c|c|c|c|c|c|c|c|c|c|}
\hline \multirow{3}{*}{ Escalas } & & \multicolumn{4}{|c|}{ Acorde con la teoría AT } & \multicolumn{4}{|c|}{ No acorde con la teoría $C T$} & \multicolumn{4}{|c|}{$\begin{array}{c}\text { Con valores diferenciados } \\
\text { por sexo }\end{array}$} \\
\hline & & \multicolumn{2}{|c|}{ Hombres } & \multicolumn{2}{|c|}{ Mujeres } & \multicolumn{2}{|c|}{ Hombres } & \multicolumn{2}{|c|}{ Mujeres } & \multicolumn{2}{|c|}{ Hombres } & \multicolumn{2}{|c|}{ Mujeres } \\
\hline & & Cat & $\mathrm{PD}$ & Cat & $\mathrm{PD}$ & Cat & $\mathrm{PD}$ & Cat & PD & Cat & PD & Cat & PD \\
\hline 1 & Introvertido & AT & $* * *$ & AT & $* * *$ & & & & & & & & \\
\hline $2 \mathrm{~A}$ & Inhibido & $\mathrm{AT}$ & $* * *$ & $\mathrm{AT}$ & $* * *$ & & & & & & & & \\
\hline $2 \mathrm{~B}$ & Afligido & AT & $* * *$ & AT & $* * *$ & & & & & & & & \\
\hline 3 & Sumiso & & & & & $\mathrm{CT}$ & $*$ & $\mathrm{CT}$ & $*$ & & & & \\
\hline 4 & Dramatizador & & & & & $\mathrm{CT}$ & $* *$ & $\mathrm{CT}$ & $* * *$ & & & & \\
\hline 5 & Egoísta & & & & & $\mathrm{CT}$ & $* * *$ & $\mathrm{CT}$ & $* * *$ & & & & \\
\hline $6 \mathrm{~A}$ & Trasgresor & AT & $* * *$ & AT & $* * *$ & & & & & & & & \\
\hline $6 \mathrm{~B}$ & Poderoso & AT & $* * *$ & AT & $* * *$ & & & & & & & & \\
\hline 7 & Conformista & & & & & $\mathrm{CT}$ & $* * *$ & $\mathrm{CT}$ & $* * *$ & & & & \\
\hline $8 \mathrm{~A}$ & Oposicionista & AT & $* * *$ & AT & $* * *$ & & & & & & & & \\
\hline $8 \mathrm{~B}$ & Autodegradante & AT & $* * *$ & AT & $* * *$ & & & & & & & & \\
\hline 9 & Tendencia Limítrofe & $\mathrm{AT}$ & $* * *$ & $\mathrm{AT}$ & $* * *$ & & & & & & & & \\
\hline A & Difusión de la Identidad & $\mathrm{AT}$ & $* * *$ & $\mathrm{AT}$ & $* * *$ & & & & & & & & \\
\hline $\mathrm{B}$ & Autodevaluación & AT & $* * *$ & $\mathrm{AT}$ & $* * *$ & & & & & & & & \\
\hline $\mathrm{C}$ & Desaprobación Corporal & AT & $* * *$ & AT & $* * *$ & & & & & & & & \\
\hline $\mathrm{D}$ & Incomodidad Sexual & & & & & $\mathrm{CT}$ & $* * *$ & $\mathrm{CT}$ & $* * *$ & & & & \\
\hline $\mathrm{E}$ & Inseguridad Grupal & $\mathrm{AT}$ & $* *$ & $\mathrm{AT}$ & $* * *$ & & & & & & & & \\
\hline $\mathrm{F}$ & Insensibilidad Social & & & & & & & & & AT & $*$ & ND & \\
\hline G & Discordia Familiar & AT & $* * *$ & AT & $* * *$ & & & & & & & & \\
\hline $\mathrm{H}$ & Abuso Infantil & AT & $* * *$ & $\mathrm{AT}$ & $* * *$ & & & & & & & & \\
\hline AA & Disfunciones Alimentarias & AT & $* * *$ & AT & $* * *$ & & & & & & & & \\
\hline $\mathrm{BB}$ & Tend. Abuso Sustancias & AT & $* * *$ & AT & $* * *$ & & & & & & & & \\
\hline $\mathrm{CC}$ & Predispos. Delincuencia & & & & & & & & & AT & $* * *$ & ND & \\
\hline DD & Tendencia Impulsividad & AT & $* * *$ & AT & $* * *$ & & & & & & & & \\
\hline $\mathrm{EE}$ & Sentimientos de Ansiedad & & & & & & & & & $\mathrm{CT}$ & $* * *$ & ND & \\
\hline $\mathrm{FF}$ & Afecto Depresivo & AT & $* * *$ & AT & $* * *$ & & & & & & & & \\
\hline GG & Tendencia Suicida & AT & $* * *$ & AT & $* * *$ & & & & & & & & \\
\hline
\end{tabular}

Nota. Cat $=$ Categoría de escala, $\mathrm{PD}=$ Poder de discriminación según $A z$, AT = Acorde con la teoría, $\mathrm{CT}=$ Contra-teórica, $\mathrm{ND}=$ No diferenciadora.

$* * * p<.001 * * p<.01 * p<.05$ 
La integración de la información comentada indica que las escalas MACI pueden, efectivamente, ser clasificadas en tres grupos tal como lo proponían Vinet y Forns (2006):

(1) El primer grupo está compuesto por aquellas escalas que discriminan apropiadamente y que se comportan de acuerdo con la teoría de Millon; en ellas se observan puntajes medios mayores en el grupo de consultantes y curvas ROC con capacidad de discriminación adecuada. A este grupo se le ha denominado escalas Acordes con la Teoría (AT).

(2) El segundo grupo está compuesto por escalas que discriminan apropiadamente y que se comportan al contrario de lo esperado teóricamente; en ellas se observa que los puntajes medios mayores corresponden al grupo de no-consultantes y que las curvas ROC tienen capacidad de discriminación adecuada. A este grupo se le ha denominado escalas Contra-Teóricas (CT).

(3) El último grupo, presente sólo en las mujeres, está formado por escalas que no poseen capacidad para diferenciar entre no-consultantes y consultantes; sus puntajes medios no presentan diferencias significativas, sus distribuciones se superponen y sus curvas ROC están en la diagonal de no discriminación. A ellas se las ha denominado escalas No Diferenciadoras (ND).

En la Tabla 5 se presenta un resumen con la categorización de las escalas señalando, además, su poder de discriminación en términos del valor $p$ del área bajo la curva ROC.

\section{Determinación del puntaje de anclaje central para los baremos de las escalas AT y CT}

En las escalas acordes con la teoría y contra-teóricas, utilizando como antecedente los resultados del análisis ROC y los puntajes de corte seleccionados por Vinet y Forns (2006), se determinó para cada escala el puntaje directo que mejor discriminara la pertenencia del sujeto al grupo de no-consultantes o consultantes. Con este objetivo se calcularon, para tres puntos específicos ubicados en la zona de la curva de mayor discriminación de cada escala, los índices de sensibilidad (S) y especificidad (E) y los valores complementarios MC y VG. Los dos primeros dan cuenta de la probabilidad de que un sujeto sea correctamente clasificado como consultante (S) o no-consultante (E), en tanto que $\mathrm{MC}$ y VG representan respectivamente los cómputos de malas clasificaciones y de aciertos totales correspondientes a cada puntaje elegido (Pita \& Pertegas, 2003).

Para elegir, entre los tres puntos posibles, el puntaje de corte que se utilizaría como punto de anclaje central en la baremación de cada escala, se buscó un puntaje de equilibrio que representara una buena $\mathrm{S}$ sin aumentar en demasía las clasificaciones erróneas de no-consultantes (E). Complementariamente, se utilizaron los indicadores de malas clasificaciones y el valor global privilegiando el puntaje que presentara el mayor número de aciertos. El puntaje de corte elegido para anclaje se presenta en la Tabla 6 con los indicadores S y E correspondientes para hombres y mujeres.

La decisión de establecer en cada escala un puntaje de corte que privilegie levemente la sensibilidad por sobre la especificidad se fundamenta en una evaluación de costo y beneficio respecto de la detección de adolescentes con problemas psicológicos en el contexto nacional, privilegiando la posibilidad de brindar atención psicológica a todos los adolescentes que eventualmente pudieran requerirlo.

Como ya se ha señalado los puntajes de corte fueron validados empíricamente empleando el MMPI-A como criterio externo. Dado que el procedimiento utilizado, además de validar cada puntaje de corte, aportó una gran cantidad de información inédita sobre la relación entre los dos instrumentos, se ha optado por presentar esos resultados en otro artículo que permita abordar el tema con la profundidad debida.

\section{Desarrollo de baremos para las escalas con puntajes de corte}

El puntaje directo de corte de cada una de las escalas AT y CT fue hecho equivalente a un puntaje de anclaje central igual a 50 en una escala transformada de 100 puntos, definida por un puntaje 1 como mínimo posible y un puntaje 100 como máximo posible. El cálculo de los Puntajes Transformados (PT) correspondientes a los puntajes menores y mayores al punto de corte se realizó utilizando la Fórmula 1 de trasformación lineal de puntajes entre diferentes escalas (Aiken, 1987) y se presentó en tablas de equivalencias entre puntajes directos y Puntajes Transformados.

Para facilitar la interpretación de puntajes individuales, los baremos incluyen tres puntos de referencia. Para cada escala AT, se ofrece un valor de corte centrado en el valor 50 (Puntaje Transformado o PT 50) que representa el puntaje de corte entre no-consultantes y consultantes; un valor PT inferior a 50, que indica el valor de la mediana para los grupos de no-consultantes, y un valor PT superior a 50, que indica el valor de la mediana para los consultantes. Así, además de atribuir a cada puntuación directa un valor en la escala lineal transformada, los tres puntos de referencia permiten interpretar la cercanía o alejamiento de un puntaje específico en relación al puntaje de corte y a las medianas de los grupos de no-consultantes y consultantes de la muestra de baremación. En las escalas CT se ofrecen los mismos puntos de referencia, pero en este caso la valoración del Puntaje Transformado de cada escala se invierte; los puntajes directos más altos, que corresponden a funcionamiento sano, se ubican en la parte inferior de la escala PT y los puntajes directos más bajos, 
Tabla 6. Sensibilidad y especificidad para los puntajes de corte de las escalas MACI según sexo.

\begin{tabular}{|c|c|c|c|c|c|c|c|}
\hline \multirow{2}{*}{\multicolumn{2}{|c|}{ Escalas }} & \multicolumn{3}{|c|}{ Hombres } & \multicolumn{3}{|c|}{ Mujeres } \\
\hline & & $\mathrm{PC}$ & $\mathrm{S}$ & E & PC & $\mathrm{S}$ & E \\
\hline 1 & Introvertido & 24 & 68,75 & 66,15 & 25 & 77,30 & 74,88 \\
\hline $2 \mathrm{~A}$ & Inhibido & 19 & 63,41 & 63,64 & 24 & 75,94 & 70,94 \\
\hline 2B & Afligido & 11 & 74,40 & 70,35 & 14 & 82,98 & 75,74 \\
\hline 3 & Sumiso & 48 & 57,00 & 50,25 & 54 & 55,08 & 51,49 \\
\hline 4 & Dramatizador & 42 & 58,37 & 49,00 & 36 & 71,66 & 68,29 \\
\hline 5 & Egoísta & 37 & 64,39 & 58,00 & 31 & 68,48 & 66,83 \\
\hline $6 \mathrm{~A}$ & Trasgresor & 31 & 69,27 & 68,50 & 25 & 68,98 & 65,69 \\
\hline 6B & Poderoso & 11 & 65,71 & 64,00 & 8 & 74,32 & 65,37 \\
\hline 7 & Conformista & 48 & 74,29 & 64,82 & 50 & 79,26 & 65,69 \\
\hline $8 \mathrm{~A}$ & Oposicionista & 20 & 73,81 & 65,15 & 20 & 82,01 & 76,85 \\
\hline $8 \mathrm{~B}$ & Autodegradante & 17 & 73,30 & 69,04 & 23 & 81,08 & 75,86 \\
\hline 9 & Tendencia Limítrofe & 11 & 72,17 & 71,00 & 13 & 79,89 & 68,14 \\
\hline A & Difusión de la Identidad & 14 & 71,23 & 68,00 & 15 & 75,13 & 69,90 \\
\hline B & Autodevaluación & 16 & 77,25 & 69,39 & 26 & 75,40 & 74,63 \\
\hline $\mathrm{C}$ & Desaprobación Corporal & 5 & 66,04 & 52,02 & 14 & 67,20 & 66,02 \\
\hline $\mathrm{D}$ & Incomodidad Sexual & 29 & 62,56 & 62,12 & 34 & 63,10 & 58,33 \\
\hline $\mathrm{E}$ & Inseguridad Grupal & 8 & 58,77 & 50,25 & 9 & 67,20 & 57,64 \\
\hline $\mathrm{F}$ & Insensibilidad Social & 29 & 60,48 & 55,50 & \multicolumn{3}{|c|}{ Escala sin puntaje de corte } \\
\hline G & Discordia Familiar & 14 & 69,19 & 63,00 & 15 & 76,60 & 67,48 \\
\hline $\mathrm{H}$ & Abuso Infantil & 5 & 78,95 & 64,65 & 8 & 80,54 & 71,84 \\
\hline AA & Disfunciones Alimentarias & 6 & 60,10 & 62,12 & 17 & 69,31 & 65,85 \\
\hline BB & Tendencia Abuso Sustancias & 13 & 76,19 & 66,50 & 10 & 75,27 & 71,71 \\
\hline $\mathrm{CC}$ & Predisposición Delincuencia & 25 & 62,32 & 62,63 & \multicolumn{3}{|c|}{ Escala sin puntaje de corte } \\
\hline DD & Tendencia a la Impulsividad & 17 & 68,10 & 62,00 & 14 & 66,49 & 61,95 \\
\hline EE & Sentimientos de Ansiedad & 29 & 57,42 & 57,07 & \multicolumn{3}{|c|}{ Escala sin puntaje de corte } \\
\hline FF & Afecto Depresivo & 12 & 70,62 & 64,82 & 21 & 73,54 & 73,17 \\
\hline GG & Tendencia Suicida & 5 & 76,30 & 73,00 & 8 & 81,38 & 73,04 \\
\hline
\end{tabular}

Nota. $\mathrm{PC}=$ Punto de corte; $S=$ Sensibilidad; $E=$ Especificidad

correspondientes a funcionamiento alterado, se ubican en la parte superior de la escala PT.

En la Tabla 7 se presentan las medianas de no-consultantes y consultantes y sus correspondientes Puntajes Transformados para hombres y mujeres. Las tablas de baremos con las puntuaciones directas y la Puntuaciones Transformadas de cada escala in extenso están disponibles y pueden ser solicitadas a la autora principal de este estudio.

\section{Desarrollo de baremos para las escalas no diferen- ciadoras y modificadoras}

Para baremar las tres escalas no diferenciadoras presentes en las mujeres (Insensibilidad Social, Predisposición a la Delincuencia y Sentimientos de Ansiedad; F, CC y EE), y las escalas modificadoras (Sinceridad, Deseabilidad y
Depreciación; X, Y y Z) de hombres y mujeres, se utilizó una estrategia que consistió en considerar la muestra completa, separada por sexo, y realizar una transformación percentilar. Esta alternativa, que sortea la ausencia de puntaje de corte, permite utilizar la información de la muestra de baremación como referente para la interpretación de estas escalas en los adolescentes evaluados con el MACI. Sus puntuaciones directas y las puntuaciones transformadas a percentiles también están disponibles en las tablas baremos que pueden ser solicitadas a la autora principal de este estudio.

\section{Discusión}

El objetivo principal de este estudio fue desarrollar baremos para el MACI en una población chilena. La ausencia 
Tabla 7. Puntajes de referencia y sus equivalencias en Puntajes Transformados en los baremos de hombres y mujeres.

\begin{tabular}{|c|c|c|c|c|c|c|c|c|c|}
\hline & & \multicolumn{4}{|c|}{ Hombres } & \multicolumn{4}{|c|}{ Mujeres } \\
\hline \multicolumn{2}{|c|}{ Escalas } & \multicolumn{2}{|c|}{ No-consultantes } & \multicolumn{2}{|c|}{ Consultantes } & \multicolumn{2}{|c|}{ No-consultantes } & \multicolumn{2}{|c|}{ Consultantes } \\
\hline & & Md & PT & Md & PT & Md & PT & Md & PT \\
\hline 1 & Introvertido & 20 & 42 & 27 & 53 & 19 & 38 & 33 & 59 \\
\hline $2 \mathrm{~A}$ & Inhibido & 17 & 45 & 23 & 54 & 18 & 38 & 33 & 60 \\
\hline $2 \mathrm{~B}$ & Afligido & 7 & 32 & 18 & 59 & 8 & 29 & 26 & 67 \\
\hline 3 & Sumiso & 49 & 49 & 46 & 52 & 55 & 48 & 52 & 52 \\
\hline 4 & Dramatizador & 43 & 48 & 40 & 52 & 40 & 43 & 31 & 57 \\
\hline 5 & Egoísta & 39 & 46 & 34 & 54 & 36 & 43 & 26 & 58 \\
\hline $6 \mathrm{~A}$ & Trasgresor & 27 & 44 & 37 & 57 & 21 & 42 & 30 & 55 \\
\hline $6 \mathrm{~B}$ & Poderoso & 9 & 41 & 14 & 54 & 6 & 38 & 13 & 57 \\
\hline 7 & Conformista & 51 & 41 & 42 & 56 & 53 & 46 & 41 & 59 \\
\hline $8 \mathrm{~A}$ & Oposicionista & 16 & 40 & 28 & 58 & 13 & 33 & 29 & 58 \\
\hline $8 \mathrm{~B}$ & Autodegradante & 10 & 30 & 25 & 57 & 14 & 31 & 37 & 64 \\
\hline 9 & Tendencia Limítrofe & 8 & 37 & 16 & 59 & 10 & 39 & 21 & 65 \\
\hline A & Difusión de la Identidad & 10 & 36 & 18 & 56 & 10 & 34 & 21 & 59 \\
\hline $\mathrm{B}$ & Autodevaluación & 11 & 35 & 25 & 59 & 15 & 29 & 39 & 66 \\
\hline $\mathrm{C}$ & Desaprobación Corporal & 4 & 34 & 7 & 52 & 10 & 36 & 18 & 61 \\
\hline $\mathrm{D}$ & Incomodidad Sexual & 31 & 47 & 27 & 53 & 36 & 46 & 33 & 51 \\
\hline $\mathrm{E}$ & Inseguridad Grupal & 7 & 44 & 9 & 52 & 8 & 45 & 13 & 58 \\
\hline $\mathrm{F}$ & Insensibilidad Social & 27 & 47 & 30 & 51 & \multicolumn{4}{|c|}{ Escala sin puntaje de corte } \\
\hline G & Discordia Familiar & 12 & 43 & 19 & 58 & 12 & 40 & 20 & 59 \\
\hline $\mathrm{H}$ & Abuso Infantil & 3 & 30 & 8 & 55 & 5 & 32 & 13 & 59 \\
\hline $\mathrm{AA}$ & Disfunciones Alimentarias & 4 & 34 & 8 & 53 & 11 & 33 & 23 & 61 \\
\hline $\mathrm{BB}$ & Tendencia Abuso Sustancias & 9 & 35 & 19 & 56 & 6 & 30 & 15 & 55 \\
\hline $\mathrm{CC}$ & Predisposición Delincuencia & 23 & 46 & 27 & 54 & \multicolumn{4}{|c|}{ Escala sin puntaje de corte } \\
\hline DD & Tendencia a la Impulsividad & 14 & 41 & 20 & 57 & 12 & 43 & 18 & 58 \\
\hline $\mathrm{EE}$ & Sentimientos de Ansiedad & 31 & 48 & 28 & 53 & \multicolumn{4}{|c|}{ Escala sin puntaje de corte } \\
\hline $\mathrm{FF}$ & Afecto Depresivo & 10 & 42 & 18 & 57 & 14 & 34 & 29 & 61 \\
\hline GG & Tendencia Suicida & 2 & 21 & 9 & 56 & 3 & 19 & 17 & 64 \\
\hline
\end{tabular}

Nota. $M d=$ Mediana; PT $=$ Puntaje Transformado

de datos de prevalencia psicopatológica para adolescentes chilenos y la necesidad de contar con un instrumento que permitiera el screening psicopatológico en población comunitaria, obligó a plantear una forma de baremación que se ha apartado notoriamente de la baremación original realizada por Millon (1993).

La escala de medida con puntajes de corte, adoptada como aspecto relevante de la baremación que se presenta, surge como respuesta a esta doble problemática. Por una parte soslaya la ausencia de datos de prevalencia de trastornos de la salud mental en adolescentes chilenos y por otra descarta las alternativas tradicionales de escalas estandarizadas (percentiles y puntajes T) por la dificultad de representar en ellas a los dos grupos que aportan información sobre el continuo salud-alteración de los adolescentes. Mantener el poder clasificatorio que ofrece el punto de corte y combinar esta información con la dimensionalidad de las conductas sanas o alteradas de los dos grupos seleccionados supone una decisión que puede ser, a la par, la más controvertida y la de mayor originalidad de este trabajo.

Esta decisión ha sido, a la luz de los resultados obtenidos, altamente pertinente. Esta muestra de baremación no tradicional, dicotomizada según procedencia (no-consultantes y consultantes) entrega resultados compatibles con los de otros trabajos previos elaborados también en muestras de consultantes y no-consultantes (Vinet \& Alarcón, 2003a; Alarcón et al., 2005; Vinet \& Forns, 2006), estableciendo así una continuidad entre los estudios.

Además, los análisis estadísticos para determinar los puntajes de corte entregaron, en cada etapa, información sobre las características y comportamiento de las escalas del MACI que van más allá del puntaje de corte mismo. 
En las escalas capaces de diferenciar entre no-consultantes y consultantes se conoce la dirección de la diferenciación (AT o CT) y el poder de discriminación específico de cada escala; esta información proporciona una apreciación global del comportamiento de las escalas clínicas del MACI en el grupo estudiado. Por su parte, los análisis de sensibilidad y especificidad para el puntaje de corte elegido muestran la real capacidad de discriminación de dicho punto y la probabilidad de tener falsos positivos o falsos negativos al diagnosticar según su valor. Finalmente, la información que entrega el puntaje de corte como puntaje central de la escala de baremación (PT 50) es complementada por el valor que adquieren, en la escala PT, las medianas de los no-consultantes y consultantes respectivamente. El uso de estos tres puntos de referencia, además de los puntajes transformados del resto de las puntuaciones directas, permite una interpretación de los datos individuales en términos de la pertenencia o no del sujeto evaluado a un grupo clínico y de cuan leve o grave es la alteración, en el caso de existir.

En el ámbito psicométrico, al término de este trabajo podemos señalar que se abre una posibilidad de integración de dos sistemas de medida que frecuentemente son vistos como contradictorios. El sistema de baremación que se presenta en este trabajo aúna criterios categoriales a través del puntaje de corte diferenciador entre normalidad y patología y criterios dimensionales a través de la selección de diversos puntos de referencia, tales como son los valores de las medianas de los dos grupos.

En el ámbito de la evaluación clínica aplicada es importante destacar que la categorización de las escalas MACI en tres tipos (acordes con la teoría, contra-teóricas y no diferenciadoras) indica que el MACI no se comporta en población chilena de la misma forma que en la población original.

Particularmente, este estudio corrobora el comportamiento contra-teórico de algunas escalas reportado inicialmente por Blumentritt et al. (2004) en muestras hispanas y que fue analizado en Chile por Vinet y Forns (2006) utilizando una interpretación transcultural centrada en la relevancia de la dimensión individualismo-colectivismo (Hofstede, 1980, Triandis, 2001) en variables de personalidad en contextos culturales latinoamericanos. Al respecto, el estudio actual indica que las características personales relacionadas con el colectivismo (expresión de los afectos, sociabilidad, dependencia del grupo de pares, respeto a las reglas y al grupo de pertenencia) se reflejan en las puntuaciones directas más elevadas que los adolescentes chilenos no-consultantes -por lo tanto, saludables y adaptados- obtienen en las escalas de patrones de la personalidad vinculadas a la interacción social (Sumiso, Dramatizador, Egoísta y Conformista).

Por su parte, la orientación contra-teórica de la escala Incomodidad Sexual está relacionada con una distribución bimodal de las puntuaciones de los no-consultantes, ella indica que este grupo de jóvenes está compuesto tanto por adolescentes con un manejo de la sexualidad adecuado y acorde a la edad como por adolescentes que experimentan grandes contradicciones y confusión en esta área. Lo anterior puede ser atribuido a características actuales de nuestra sociedad donde los temas vinculados a la sexualidad generan confusión y conflicto no sólo en los adolescentes sino que también en la sociedad adulta.

Por otro lado, la presencia en la muestra de mujeres, de tres escalas sin capacidad de discriminación entre noconsultantes y consultantes puede entenderse a través de diferentes hipótesis alternativas o complementarias. Una de ellas señala aspectos comunes en la experiencia de las adolescentes con y sin trastornos en los ámbitos de evaluación de las escalas involucradas (interacción social trasgresora y sentimientos de ansiedad); otra alternativa indica una escasa diferenciación de la muestra de baremación al no contar con un número suficiente de adolescentes mujeres con problemas de desadaptación social y conductas delictivas; una tercera alternativa señalaría, tal como proponen Vinet y Forns (2006) para la escala de ansiedad, una construcción psicométrica débil.

Esta última alternativa, referida a la escala de ansiedad (EE), se ve fortalecida por el comportamiento contra-teórico que esta escala presenta en la muestra de varones. En tanto no se aclare su comportamiento psicométrico, nuestra recomendación es prescindir de la información que esta escala pudiera aportar tanto en términos de presencia como de ausencia de ansiedad.

Los interrogantes que surgen con la presencia de las escalas contra-teóricas y no diferenciadoras en los adolescentes chilenos, han sido estudiadas más a fondo en el trabajo que da origen a la baremación chilena del MACI; ellas y sus respuestas tentativas exceden los objetivos de este artículo orientado sólo a dar a conocer las características más relevantes del proceso de baremación del MACI; sin embargo, tenemos claro que el objetivo general de la baremación sólo será alcanzado en plenitud cuando se pueda proporcionar interpretaciones adecuadas para las puntuaciones que los adolescentes chilenos obtienen en el MACI, sobretodo al constatar que el contexto cultural de nuestro país permeabiliza la expresión de algunos de los constructos desarrollados en el modelo de personalidad de Millon.

\section{Referencias}

Aiken, L. R. (1987). Formulas for equating ratings on different scales. Educational and Psychological Measurement, 47, 51-54.

Alarcón, P., Vinet, E., \& Salvo, S. (2005). Estilos de personalidad y desadaptación social durante la adolescencia. PSYKHE, 14(1), 3-16.

American Psychiatric Association. (1995). Manual diagnóstico y estadístico de los trastornos mentales: DSM- $I V$. Barcelona: Masson.

Blumentritt, T., Angle, R. \& Brown, J. (2004). MACI personality patterns and DSM-IV symptomology in a sample of troubled Mexican-American adolescents. Journal of Child and Family Studies, 13 (2), 163-178. 
Blumentritt, T., \& Wilson, C. (2004). The Millon Adolescent Clinical Inventory: Is it valid and reliable for Mexican-American youth? Journal of Personality Assessment, 83(1), 64-74.

Butcher, J.N., Williams, C. L., Graham, J. R., Archer, R. P., Tellegen, A., Ben-Porath, Y. S. \& Kaemmer, B. (1992). MMPI-A: Manual for administration, scoring and interpretation. Minneapolis: University of Minnesota Press.

Camara, W. J., Nathan, J. S., \& Puente, A. E. (2000). Psychological test usage: Implications in professional psychology. Professional Psychology: Research and Practice, 31(2), 141-154.

Casullo, M., \& Castro, A. (2002). Patrones de personalidad, síndromes clínicos y bienestar psicológico en adolescentes. Revista de Psicopatología y Psicología Clínica, 7(2), 129-140.

Casullo, M., Góngora, V., \& Castro, A. (1998). La adaptación del inventario MACI (Millon Adolescent Clinical Inventory). Un estudio preliminar con estudiantes adolescentes argentinos. Investigaciones en Psicología, 3(2), 73-89.

Cohen, J. (1988). Statistical power analysis for the behavioral science. (2d ed.) Hillsdale NJ. Lawrence Erlbraum.

Díaz, C. (2003). Desarrollo de una tipología de adolescentes consultantes en salud mental, a partir del Inventario Clínico para Adolescentes de Millon. Tesis de Magíster en Psicología, Universidad de La Frontera, Chile.

Hofstede, G. (1980). Culture's consequences: International differences in work related value. Beverly Hills: Sage.

Hsu, L. M. (2002). Diagnostic validity statistic and the MCMI-III. Psychological Assessment, 14(4), 410-422.

Iza, M. (2002). Personalidad, adolescencia y delito. Un estudio en adolescentes institucionalizados de Lima-Perú. Tesis de Master Universitario en Evaluación Psicológica Clínica y Forense, Universidad de Salamanca, España.

Meeker, L. (2002). Adolescent attachment and prediction of problematic personality styles. Dissertation Abstracts International: Section B: The Sciences and Ingineering, 63, 5B, 2595.

Metz, C. E. (1998). ROCKIT 0.9B. Beta Version. Universidad de Chicago. Documento de Internet recuperado el 25 de Octubre de 2004 desde http://xray.bsd.uchicago.edu/krl/roc soft.htm.

Millon, T. (1969). Modern psychopathology: A biosocial approach to maladaptative learning and functioning. Philadelphia: Saunders.

Millon, T. (1990). Toward a new personology. An evolutionary model. New York: Wiley.

Millon, T. (1993). Manual of Millon Adolescent Clinical Inventory. Minneapolis: Nacional Computer Systems.
Pérez, M. V., Díaz, A. \& Vinet, E. (2005). Características psicológicas de adolescentes pertenecientes a comunidades educativas vulnerables. Psicothema, 17(1), 37-42.

Pita, S., \& Pértegas, S. (2003). Pruebas diagnósticas. Cadena de Atención Primaria, 10, 120-124. Documento de Internet recuperado el 20 de Octubre de 2004 desde http:/www.fisterra.com/mbe/investiga/pruebas_diagnosticas/pruebas_diagnosticas.htm.

TEA Ediciones. (2004). MACI. Inventario Clínico para Adolescentes de Millon. Manual. Adaptación española de Gloria Aguirre Llagostera. Madrid: Autor.

Triandis, H. C. (2001). Individualism-Collectivism and personality. Journal of Personality, 69(6), 907-924.

Vinet, E., \& Alarcón, P. (2003a). El Inventario Clínico para Adolescentes de Millon (MACI) en la evaluación de adolescentes chilenos. PSYKHE, 1(1), 39-55.

Vinet, E., \& Alarcón, P. (2003b). Evaluación psicométrica del Inventario Multifásico de la Personalidad de Minnesota para Adolescentes (MMPI-A) en muestras chilenas. Terapia Psicológica, 21(2), 87-103.

Vinet, E., Brió, C., Correa, P., Díaz, P., Diez, M., Echeverría, M., Salazar, D., \& Vargas, A. (1999). MACI. Traducción y adaptación chilena para uso exclusivo en investigación. Proyecto DIDUFRO 9966. Universidad de La Frontera, Temuco, Chile.

Vinet, E. \& Forns, M. (2006). El Inventario Clínico para Adolescentes de Millon (MACI) y su capacidad para discriminar entre población general y clínica. PSYKHE, 15(2), 69-80.

Vinet, E., González, M E. \& Alarcón, P. (2002, Octubre). MACI in Chile: A study with two groups presenting psychological problems. Trabajo presentado en The Ninth Annual Millon Clinical Inventories Conference. Minneapolis - USA.

Vinet, E.; González, M. E.; Alarcón, P., Salvo, S., Pérez, V. \& Díaz, A (2004). Personalidad y psicopatología en adolescentes: Perfiles diferenciales en tres muestras chilenas y estudio de validez transcultural de los instrumentos utilizados. Informe Final. Proyecto FONDECYT 1010514, Universidad de La Frontera, Temuco, Chile.

Vinet, E. \& Lucio, M. E. (2005). Aplicabilidad de las normas mexicanas y estadounidenses del MMPI-A en la evaluación de adolescentes chilenos. Revista Mexicana de Psicología, 22(2), 519-528.

Vinet, E., Salvo, S. \& Forns, M. (2005). Adolescentes no-consultantes en riesgo: Una evaluación a través del MACI. Anuario de Psicología, 36(1), 83-97.

Wasserman, A. H. (2001). Exploring normal adolescent sex offenders: Investigating moral rigidity. Dissertation Abstracts International: Section B: The Sciences and Ingineering, 61(11-B), 6122. 